\title{
Traduire
}

Une eutre perspective sur $r$ tatadciction

Revue française de la traduction

$244 \mid 2021$

Des jeux et des mots

\section{La traduction de jeux vidéo en interne}

\section{Géraldine Oudin}

\section{(2) OpenEdition}

Journals

Édition électronique

URL : https://journals.openedition.org/traduire/2283

DOI : 10.4000/traduire.2283

ISSN : 2272-9992

\section{Éditeur}

Société française des traducteurs

Édition imprimée

Date de publication : 15 juin 2021

Pagination : 22-26

ISSN : 0395-773X

\section{Référence électronique}

Géraldine Oudin, «La traduction de jeux vidéo en interne », Traduire [En ligne], 244 | 2021, mis en ligne le 15 juin 2021, consulté le 01 juillet 2021. URL : http://journals.openedition.org/traduire/2283 ; DOI : https://doi.org/10.4000/traduire.2283 


\section{La traduction de jeux vidéo en interne}

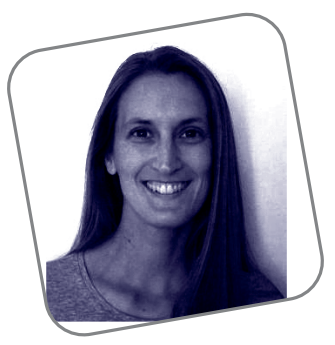

\section{Géraldine Oudin}

Pendant plusieurs années, j'ai eu pour client un célèbre studio de jeux vidéo qui faisait appel à des traducteurs indépendants afin de renforcer son équipe plusieurs mois par an. C'est cette expérience positive que je me propose de raconter ici, en abordant différents aspects.

\section{Le début de l'aventure}

Tout a commencé en Australie, à l'automne 2011. À la suite de l'accident nucléaire de Fukushima, j'ai renoncé à retourner au Japon comme prévu après mon séjour à Perth. L'antenne européenne d'un studio japonais me contacte avec une proposition alléchante: intégrer son équipe quelques mois, le temps de traduire en équipe un célèbre jeu vidéo. L'entreprise est très à cheval sur la confidentialité et craint les fuites, il est donc exclu de travailler à distance. Elle me propose un billet d'avion, un logement de fonction et un salaire horaire généreux. Tout cela me paraît presque trop beau pour être vrai, mais la présence d'un ami dans l'équipe permanente me rassure, et je décide de tenter l'aventure. Nous prenons 
rendez-vous pour un test de traduction de quatre heures, à l'issue duquel le projet est confirmé. J'atterris dans la capitale anglaise et je fais la connaissance de mes collègues hauts en couleur, répartis en quatre équipes en fonction de leur langue cible: français, allemand, espagnol et italien. Tous travaillent directement depuis le japonais. Dans un film comme Les traducteurs (Regis Roinsard, 2018), c'est à ce moment-là que tout commence à déraper, mais comme le monde des études japonaises est très petit, je découvre que j'ai déjà croisé tous les membres de l'équipe à un moment ou à un autre de mon cursus, et qu'ils sont tous à peu près équilibrés.

\section{La confidentialité avant tout}

Dans un souci de confidentialité, nous travaillons uniquement dans les locaux du client, dont l'accès est sécurisé, sur les ordinateurs qui sont mis à notre disposition. Pour des raisons évidentes, rien ne doit sortir de l'entreprise. Les ROM mises à notre disposition sont sous clé dans une salle à part. II y a des persiennes aux fenêtres. On nous met en garde contre les éventuels fans qui pourraient tenter de nous soutirer des informations.

\section{Le travail en équipe}

Lors de cette première mission, je n'ai encore que trois ans et demi d'expérience. Jusque-là, j'ai principalement travaillé pour des agences de traduction qui ont rarement pris la peine de commenter mon travail, en bien comme en mal. Je m'efforce de fournir un service de qualité et je suppose que mes clients sont satisfaits puisqu'ils reviennent vers moi, mais je n'ai pas de véritable retour, en dehors de celui des ONG pour lesquelles je fais parfois du bénévolat.

En revanche, dans une société de jeu vidéo, le travail d'équipe est de mise, et c'est un aspect qui va beaucoup m'apporter: je prends conscience de mes tics de langage et je m'efforce d'y remédier. Je réalise que chacun a son style et ses points forts, mais que personne n'est infaillible. Cerise sur le gâteau, nous pouvons nous permettre d'être malades (mais pas tous en même temps). Nous nous relisons 
mutuellement, puis les fichiers sont confiés à des éditeurs qui effectuent non seulement une vérification classique (orthographe, grammaire, syntaxe), mais lissent également la traduction et s'assurent qu'elle respecte la terminologie et la charte maison, toujours en interne. À cette étape, il y a encore de nombreux échanges entre éditeurs et traducteurs. Les testeurs professionnels quant à eux interviennent plus tard pour repérer les éventuels bugs une fois le jeu finalisé.

Concrètement parlant, pour chaque projet (d'une durée de plusieurs mois à temps plein), un traducteur interne expérimenté endosse le rôle de chef de projet. C'est cette personne qui répartit les fichiers au mieux entre les membres de l'équipe. Les premières années, nous travaillons sur un produit fini (le jeu sort dans un premier temps dans son pays d'origine avant d'être traduit en différentes langues), mais bientôt les sorties se font en simultané dans toutes les langues, ce qui signifie que le responsable doit jongler avec le matériel dont il dispose. L'exercice est périlleux, d'autant que les textes ne nous parviennent pas nécessairement dans l'ordre chronologique du jeu. Heureusement, nous avons des avantages majeurs sur nos collègues qui travaillent à distance pour d'autres entreprises, parfois pour le compte d'intermédiaires: nous avons accès aux ROM en constante évolution et à une documentation foisonnante, nous côtoyons des employés qui connaissent l'histoire de la franchise depuis la nuit des temps et nous pouvons poser toutes nos questions aux développeurs. Les vérifications et le lissage finaux sont renforcés.

Nous avons également la possibilité de réserver une salle de réunion pour nos sessions de brainstorming. Les idées fusent, nous nous renvoyons la balle jusqu'à avoir mis le doigt sur le nom parfait pour tel personnage ou tel lieu. Les trouvailles sont toujours meilleures à plusieurs.

\section{Au-delà de la traduction}

Le fait de travailler en interne a d'autres avantages, notamment l'accès à des formations spécifiques, par exemple à des outils d'aide à la traduction développés par l'entreprise et que les traducteurs ont contribué à améliorer (je me souviendrai toujours de l'arrivée des «tags», un dispositif permettant de proposer plusieurs versions d'une même phrase 
en fonction de différents critères, comme le sexe du joueur, pour citer le plus emblématique) ou à des sessions liées à l'industrie du jeu vidéo, comme sur le classement PEGl (classification par âge des jeux vidéo), qui a une grande influence sur la traduction de ce type de supports.

Enfin, les traducteurs en interne peuvent être amenés à faire office de «consultant culturel» et à donner leur avis sur des aspects non linguistiques, comme la question (récurrente) de la longueur des jupes des personnages en fonction de leur âge et de celui du public cible. C'est valorisant, car en règle générale, un indépendant n'a pas voix à ce chapitre-là. Le nom des traducteurs est bien entendu cité dans le générique, mais la plus grande reconnaissance vient des fans euxmêmes, que ce soit sur les forums ou dans la vraie vie, comme cette maman qui, dans le train, m'a confié qu'elle ne laissait son fils jover qu'à telle franchise parce que les dialogues français étaient si bien rédigés qu'il apprenait même des mots de vocabulaire.

\section{Conclusion}

La traduction de jeux vidéo en interne a de nombreux avantages et très peu d'inconvénients, en dehors d'une hiérarchie parfois lourde et de la nécessité de défendre certains choix face à des non-linguistes. Les principales difficultés sont d'ordre organisationnel, car les traducteurs sont tributaires des progrès des développeurs: la charge de travail est souvent inégalement répartie pendant un projet, avec des débuts calmes et des fins très chargées, et plus on avance dans sa carrière, moins il est facile de se libérer pour plusieurs mois à la dernière minute.

Je pense que les grands studios japonais ont bien compris l'intérêt, non seulement en termes de confidentialité, mais aussi de qualité, de faire traduire leurs jeux en interne et continueront à résister à la tentation d'externaliser une étape qui peut contribuer au succès du produit final. Les forums dédiés regorgent de témoignages qui confortent cette assertion. 
Géraldine Oudin travaille du japonais et de l'anglais vers le français. Elle traduit aujourd'hui principalement pour l'édition (fiction et non-fiction) et les institutions culturelles, mais elle a cumulé plusieurs années d'expérience à temps plein dans le domaine des jeux vidéo. Elle s'intéresse également au doublage et au sous-titrage, qui présentent des contraintes proches de la traduction de mangas et de jeux vidéo.

www.zentranslations.com 